\title{
The Potential Therapeutic Role of Proton Pump Inhibitors in COVID-19: Hypotheses Based on Existing Evidences
}

Authors

Avik Ray (D), Swati Sharma, Balakrishnan Sadasivam

Affiliation

Department of Pharmacology, All India Institute of Medical

Sciences Bhopal, Bhopal, Madhya Pradesh, India

Key words

pharmacology, molecular pharmacology, infectious

diseases, gastrointestinal pharmacology, antiviral drugs

received 26.06 .2020

accepted 01.08.2020

Published online: 2.9 .2020

\author{
Bibliography \\ Drug Res 2020; 70: 484-488 \\ DOI 10.1055/a-1236-3041 \\ ISSN 2194-9379
}

(c) 2020. Thieme. All rights reserved.

Georg Thieme Verlag KG, Rüdigerstraße 14,

70469 Stuttgart, Germany

\section{Correspondence}

Dr. Avik Ray

Department of Pharmacology, 3rd Floor, Medical College, All

India Institute of Medical Sciences Bhopal

Bhopal 462020

Madhya Pradesh

India

Tel.: + 919674359961

avik.jrpharma18@aiimsbhopal.edu.in

\section{ABSTRACT}

Although the major therapeutic uses of the proton pump inhibitors are in gastric-acid related diseases, evidences are suggestive of a pleiotropic nature of the compounds. We comment on the probable pathways and cellular machineries via which proton pump inhibitors could show beneficial therapeutic effects against SARS-CoV-2 based on the existing evidences. Proton pump inhibitors have shown antiviral potencies in various in vivo and in vitro studies. Some of the major possible ways through which they can act against SARS-CoV-2 are by exerting anti-inflammatory and anti-fibrotic effects, via vacuolar ATPase pumps leading to raised endolysosomal $\mathrm{pH}$ and by targeting endosomal complexes. The current pandemic has put forward a challenge to find treatment options. Although the potential roles of proton pump inhibitors against SARS-CoV-2 have been discussed in recent publications, the clinical evidences for their real-world effectiveness do not point towards a beneficial effect clearly yet. We suggest that although proton pump inhibitors should strongly be considered as potential therapeutic options for COVID-19, larger studies in the form of randomized controlled trials would be required to arrive at a definite conclusion.

\section{Introduction}

Proton pump inhibitors (PPIs) are one of the most widely prescribed group of drugs for the treatment of various gastric-acid associated disorders such as peptic ulcer disease and gastro-oesophageal reflux disease (GERD). They have been shown to have multiple therapeutic effects besides on gastric acid diseases. One factor which might contribute to this pleiotropic nature of the PPIs is the presence of benzimidazole scaffold in their structure, which is considered a privilege since it imparts the ability to simultaneously target various biological molecules [1].

Considering the current pandemic of COVID-19 and the necessity to find a drug for its treatment, it is of utmost importance that the antiviral potencies of the already approved drugs be explored in order to consider them for re-purposing. Since SARS-CoV-2 shows a high degree of phylogenetic similarities with previously identified coronaviruses such as SARS-CoV and MERS-CoV, it might be a possibility that drugs which show effects on these viruses may be potential candidates for screening against the novel coronavirus. We hereby explore the possibility of re-purposing PPIs against SARS-CoV-2, the pathways through which it might be possible and summarize the evidences generated till date.

\section{General antiviral properties of PPIs}

Currently, there are very few antiviral agents available for the treatment for viral infections. Conventionally used antiviral drugs such as zidovudine, acyclovir and foscarnet have shortcomings in terms of 
limited applications, serious adverse reactions and development of resistance. A patent registered in as early as 1999 by Moorman et al. showed glimpses of a potential future role of PPIs as antiviral agents by demonstrating their ability to inhibit viral serine protease [2].

\section{As an anti-inflammatory and anti-fibrotic agent}

PPIs might be associated with anti-inflammatory and antioxidative actions [3-5]. An in vitro study has shown that they can inhibit the production of pro-inflammatory cytokines such as IL-6, IL-8 and TNF- $\alpha$ [3]. Further, there are evidences to support protective role of omeprazole and lansoprazole to reduce oxidative stress in human gastric epithelial and endothelial cells [4]. Lansoprazole has been shown to reduce the number of monocytes expressing ICAM-1 in the peripheral blood [6]. Additionally, omeprazole can reduce cytokine production in duodenal epithelial cells as was observed in an in vivo study [7].

PPIs have also been associated with antiproliferative and antifibrotic properties [8]. There has been a growing rationale behind considering antifibrotic agents in COVID-19 treatment based on findings suggestive of pulmonary fibrotic diseases ranging from fibrosis related to organizing pneumonia to severe acute lung injury with massive fibrotic changes [9]. Autopsy findings have also shown pulmonary fibrosis (PF) in fatal COVID-19 cases [10]. Although it might be too early to comment on the prevalence of post-COVID PF, early analysis of patients who have been discharged from hospital is indicative of a high rate of fibrosis-associated lung function abnormalities [11].

The available antifibrotic therapies in the form of pirfenidone and nintedanib have been postulated to attenuate pro-fibrotic pathways in COVID-19. Moreover, pirfenidone has been considered for a phase 3 trial against SARS-CoV-2 [12]. Additionally, mTOR is an emerging therapeutic target in Idiopathic Pulmonary Fibrosis and PPIs have been shown to downregulate the mTOR pathway [13]. Considering the emerging concerns of PF in COVID-19, this adds on to the reasons for repurposing PPIs against SARS-CoV-2.

\section{Action on vacuolar ATPase and impact on $\mathrm{pH}$}

Located on the plasma membrane and on the surface of acidic organelles such as lysosomes and endosomes, vacuolar ATPase ( $v$-ATPase) is one of the key factors controlling vesicular $\mathrm{pH}$ [14]. It is a vacuolar-type proton pump which maintains the acidic $\mathrm{pH}$ inside organelles such as secretory granules, endosomes, lysosomes and trans-Golgi network and act as the major proton-extruding pump. It is expressed ubiquitously and performs a wide range of biological functions through vesicular, luminal and extracellular acidification [15]. Their activity could be inhibited by PPIs, leading on to cytosolic acidification and endolysosomal alkalinisation $[15,16]$.

v-ATPase mediated acidification of endosome is an important step for entry of several viruses including coronaviruses [17]. Majority of the enveloped viruses utilize the endocytic pathway leading to their fusion with the membranes of the host cell organelles such as endosomes and lysosomes. A key requirement for the normal endocytic pathway functioning is to have an acidic vesicular $\mathrm{pH}$. The viruses utilize $\mathrm{pH}$ sensing as an evolutionary strategy to monitor the endosomal maturity. Two major sensing strategies utilized by the viruses for induction of membrane fusion are direct sensing of the $\mathrm{pH}$ leading to conformational changes in the membrane glycoproteins and enzyme-mediated proteolytic cleavage [18]. Increase in this $\mathrm{pH}$ can hence lead to inhibition of viral fusion and replication. Besides, modulation of $\mathrm{pH}$ is important for exocytosis as well, leading to viral dissemination [19].

An in-vitro screening of 60 FDA approved drugs revealed antiviral potency of omeprazole, supporting its repurposing against COVID-19 [20]. Omeprazole, along with Vonoprazan (a potassium competitive acid blocker), was proved to be associated with increase in $\mathrm{pH}$ inside endosomes and golgi apparatus. The postulated hypotheses are that it happens either by blocking v-ATPase pumps or by acting as a $\mathrm{pH}$ buffer. Such modifications of $\mathrm{pH}$ would interfere with the processing of the spike (S1) protein by the endosomal proteases and restrict the egress of SARS-CoV-2 infection.

Quite interestingly, a study has shown that in cells treated with lysosomotropic agents such as $\mathrm{NH} 4 \mathrm{Cl}$ or Bafilomycin $\mathrm{A} 1$ (inhibitor of endosomal/lysosomal v-ATPase) which causes increase in the vesicular $\mathrm{pH}, \mathrm{ACE} 2$ receptors present on the cell surface get trapped within perinuclear vacuoles, suggesting that function of ACE2 might be inhibited by these agents and hence, prevent the entry of SARS-CoV-2 into the host cell [21].

Omeprazole can block the function of v-ATPase in renal cortical cells and medullary endosomes as seen in a study on rats [22]. Evidences also point towards a potential activity of lansoprazole as a v-ATPase inhibitor in gastric mucosa [23]. More recently, reports are suggestive that omeprazole and esomeprazole are capable of altering the localisation of the v-ATPase inside the cells as well [24]. This strengthens the claim about the antiviral potency of PPIs and the need for further studies regarding their possible re-purposing for COVID-19.

\section{Targeting ESCRT}

Many of the enveloped viruses utilize a host factor, the endosomal sorting complex required for transport (ESCRT), for their progression from an infected cell. Coronaviruses encode classical motifs which interact with ESCRT, one such example being P(T/S)AP [25]. This motif engages a protein Tsg101 in ESCRT-I, leading to delivery of ESCRT-III to viral budding sites, causing release of the virus particles by membrane scission. A study showed that tenatoprazole and esomeprazole, PPIs which are prodrugs, target a cysteine residue (C73) in the N-terminal domain of Tsg101, disrupting its binding with ubiquitin and preventing its localization to the plasma membrane budding site, thus inhibiting viral replication [26]. Tsg101 and ESCRT have been found to help in replication of Ebola virus [27] and Dengue virus [28] besides a few others. Like tenatoprazole and esomeprazole, other PPIs in the market also target C73 in Tsg10138, leading to a hypothesis that these compounds could be effective against a broad spectrum of viruses by inhibiting replication, including SARS-CoV-2.

\section{Learnings from COPD}

Patients who experience frequent exacerbations of chronic obstructive pulmonary disease (COPD) show high susceptibility to viral infections and poor ability to prevent their replication [29]. PPIs have shown to have impact on such viral infections (herpes virus, rhinovirus), displaying potential to prevent COPD exacerbations [30]. A randomized trial showed that lansoprazole use could 
be associated with reduction of frequency of common cold and COPD exacerbation, thus attenuating chances of contracting viral infections [31].

GERD is often found to be associated with COPD as a comorbidity, with its prevalence being higher in COPD patients as compared to healthy controls [32]. It often leads to frequent COPD exacerbation in patients. PPIs are the first-line drug of choice for managing GERD. Although there are mixed evidences, a recent nationwide study with 3,485 patients of COPD having symptomatic GERD showed that PPI use is associated with a better outcome in terms of risk of acute exacerbation of COPD and mortality [33]. By doing so, it might indirectly lead to protection of such patients against viral infections.

\section{Actions on ACE2}

SARS-CoV-2 uses ACE2 as its receptor for entry into human body. The glandular cells of gastrointestinal epithelium, mainly of the stomach, duodenum and rectum, express ACE2 protein and have been shown to exhibit the SARS-CoV-2 viral nucleocapsid protein in patients with COVID-19 [34]. ACE2 enzyme activity varies with $\mathrm{pH}$. A pH of 7-7.5 is said to be optimal for its functioning [35]. As mentioned earlier, PPIs tend to alkalinise the intraluminal environment by inhibiting v-ATPase. Since major reduction in the activities of ACE2 occurs at a pH beyond 7.5 [36], the use of PPIs which leads to a more basic $\mathrm{pH}$ [15] would render them less functional, thereby hindering the entry of SARS-CoV-2 into the cells.

ACE2 is a zinc metalloprotease and chronic PPI use is known to reduce zinc levels in the body by depleting zinc stores as well as by preventing absorption of supplemental zinc [37]. The dose of PPI required to exert anti-SARS-CoV-2 effect has been estimated to be very high. The plasma omeprazole concentration that has been shown to interfere with the cytopathogenic effects of SARS-CoV-2 $\left(\mathrm{IC}_{50} 34 \mu \mathrm{M}\right)[38]$ is far beyond its therapeutic plasma concentration of approximately $8 \mu \mathrm{M}$ required for gastric use [39]. Although not toxic, such a high systemic concentration might be associated with transient adverse drug reactions such as confusion, drowsiness, tachycardia, dry mouth, headache and hypomagnesemia but without any serious adverse outcomes [40]. Additionally, their use at such high levels could also lead to zinc deficiency despite being used for shorter duration. As per a study, omeprazole administered in therapeutic dose $(60 \mathrm{mg} /$ day) for seven days reduced the intestinal absorption of zinc and hence, its plasma levels [41]. Serum ACE activity in rats and guinea pigs has been shown to be affected adversely by zinc deficiency [42]. This is important since ACE2 exhibits substantial structural homology to ACE and is in fact also known by the name ACEH (ACE homolog).

On the contrary, presence of zinc has shown to reduce the ability of ACE2 to metabolize its substrate [43]. Its effect on the function of ACE2 as a receptor for SARS-CoV-2 is not yet clear. Moreover, other metalloproteinases like matrix metalloproteinases (MMPs) and metallothionein are also affected by zinc deficiency in mice, wherein their levels are reduced and oxidative stress response is enhanced [44].

\section{Role as a clinical add-on}

In addition to the direct antiviral effects, PPIs might also be used as an add-on to certain therapeutic agents. In an in silico study, omeprazole increased the efficacy of aprotinin, a serine protease inhibitor, and remdesivir by 2.7 -fold and 10 -fold respectively [38]. Thus, combination of aprotinin and remdesivir with omeprazole might be a potential therapy candidate for the treatment of COVID-19. Combining PPIs with non-steroidal anti-inflammatory drugs (NSAIDs) having antiviral properties such as indomethacin has also been proposed as a therapeutic option in COVID-19 [45].

\section{Clinical evidences}

It is worth mentioning that in a retrospective case-control study on 179 elderly patients, those on PPIs were 2.3 times less likely (Odds Ratio $[\mathrm{OR}]=0.4381,95 \%$ confidence interval $(\mathrm{Cl})$ [0.2331-0.8175], $\mathrm{p}=0.0053$ ) to be infected by SARS-CoV-2 and develop COVID-19 as compared to those not taking PPIs [46]. Further, an R\&D blueprint for experimental treatments, which has been developed by the World Health Organization (WHO) in order to join hands globally and accelerate the process of finding therapeutics against SARS-CoV-2, mentions the probable roles of omeprazole, lansoprazole and rabeprazole, either alone or in combinations with other drugs, for the treatment of COVID-19 [47].

However, recent evidences suggest otherwise. A populationbased online survey carried out in the United States of America revealed a dose-response relationship between intake of PPIs and getting tested as COVID-19 positive; those taking PPIs once daily $(\mathrm{OR}=2.15,95 \% \mathrm{Cl}[1.90-2.44])$ or twice daily $(\mathrm{OR}=3.67 ; 95 \% \mathrm{Cl}$ [2.93-4.60]) showed significantly higher odds for reporting a COVID-19 positive test as compared to the ones not taking PPIs [48]. Another retrospective study on 152 COVID-19 patients showed treatment with $\mathrm{PPI}$ to be associated with an increased risk of secondary infection $(\mathrm{OR}=2.37,95 \% \mathrm{Cl}$ [01.08-5.22], $\mathrm{P}=0.032$ ) and acute respiratory distress syndrome (ARDS) (48.4\% vs. $12.2 \%$, $\mathrm{P}=0.020)$ along with a significantly higher index mortality $(19.4 \%$ vs. $5.6 \%, P=0.010$ ) [49]. However, both of these studies had limitations; while the former is an observational study with possible protopathic bias, the latter is a retrospective analysis considering only hospitalized COVID-19 patients and ignoring the impact of the duration of PPI intake. Another retrospective study on 154 hospitalized adults with COVID-19 concluded on similar lines, showing that PPIs have no therapeutic role in this disease [50].

Since none of the studies could be considered as confirmatory, we need to initiate larger studies in the form of RCTs to come to a definite conclusion.

\section{Conclusions}

Although the possibilities of considering PPIs as therapeutic options in COVID-19 have been proposed and discussed in some of the recent publications $[45,51,52]$, the clinical evidence for the same is a mix bag. We have hypothesized their potential roles based on specific cellular mechanisms and summarized all the relevant findings and information till date to get a well-balanced and holistic view on the topic. Multiple pathways ( $\triangleright$ Fig. 1), as discussed, could be targeted with these drugs, leading on to disruption of the egress of COVID-19. However, the real effectiveness needs to be 


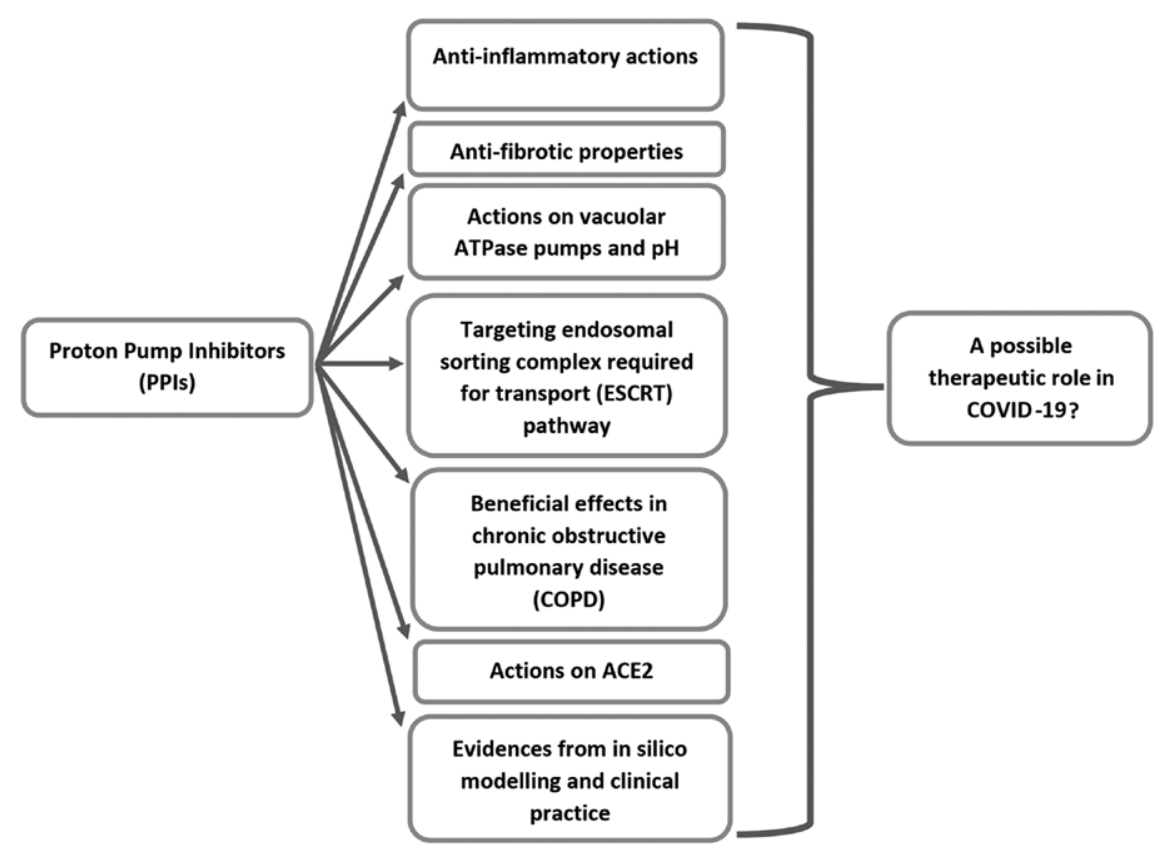

-Fig. 1 A summary of the probable mechanisms by which proton pump inhibitors (PPIs) could impart therapeutic benefits in COVID-19. While most of the hypothesized mechanisms and in silico findings support their inclusion as potential candidates for repurposing against SARS-CoV-2, clinical evidences do not point towards any benefit unambiguously, denying us from arriving at a definite conclusion.

understood through large scale pragmatic RCTs and registry-based studies to comment on the therapeutic roles of PPIs in COVID-19 with a certain degree of confidence.

\section{Funding}

This study has not received any specific grant from any funding agency in the public, commercial or not-for-profit sectors.

\section{Conflict of Interest}

The authors declare that they have no competing interest.

\section{References}

[1] Kaur G, Kaur M, Silakari O. Benzimidazoles: an ideal privileged drug scaffold for the design of multitargeted anti-inflammatory ligands. Mini Rev Med Chem 2014; 14: 747-767

[2] PubChem Database. Method of using $(\mathrm{H}+/ \mathrm{K}+)$ ATPase inhibitors as antiviral agents. 1996; https://pubchem.ncbi.nlm.nih.gov/patent/ US5945425, accessed on July 29, 2020

[3] Sasaki T, Nakayama K, Yasuda H et al. A new strategy with proton pump inhibitors for the prevention of acute exacerbations in COPD. Ther Adv Respir Dis 2011; 5: 91-103

[4] Becker JC, Grosser N, Waltke C et al. Beyond gastric acid reduction: proton pump inhibitors induce heme oxygenase- 1 in gastric and endothelial cells. Biochem Biophys Res Commun 2006; 345: 1014-1021
[5] LEGW Vanfleteren, Spruit MA, EFM Wouters, FME Franssen. Management of chronic obstructive pulmonary disease beyond the lungs. Lancet Respir Med 2016; 4: 911-924

[6] Ohara T, Arakawa T. Lansoprazole decreases peripheral blood monocytes and intercellular adhesion molecule-1-positive mononuclear cells. Dig Dis Sci 1999; 44: 1710-1715

[7] Kountouras ], Boura P, Lygidakis NJ. Omeprazole and regulation of cytokine profile in Helicobacter pylori-infected patients with duodenal ulcer disease. Hepatogastroenterology 2000; 47: 1301-1304

[8] Ghebre YT, Raghu G. Idiopathic pulmonary fibrosis: Novel concepts of proton pump inhibitors as antifibrotic drugs. Am J Respir Crit Care Med 2016; 193: 1345-1352

[9] Shi H, Han X, Jiang N et al. Radiological findings from 81 patients with COVID-19 pneumonia in Wuhan, China: a descriptive study. Lancet Infect Dis 2020; 20: 425-434

[10] Zhang T, Sun LX, Feng RE. Comparison of clinical and pathological features between severe acute respiratory syndrome and coronavirus disease 2019. 2020; 43: 496-502 Chinese DOI: 10.3760/ cma.j.cn112147-20200311-00312

[11] Mo X, Jian W, Su Z et al. Abnormal pulmonary function in COVID-19 patients at time of hospital discharge. Eur Respir J 2020; 55 (6): 2001217

[12] Zhang H. A Randomized, Open-label Study to Evaluate the Efficacy and Safety of Pirfenidone in Patients With Severe and Critical Novel Coronavirus Infection. https://clinicaltrials.gov/ct2/show/ NCT04282902, accessed on July 292020

[13] Chen M, Lu J, Wei W et al. Effects of proton pump inhibitors on reversing multidrug resistance via downregulating V-ATPases/PI3K/ Akt/mTOR/HIF-1 $\alpha$ signaling pathway through TSC1/2 complex and Rheb in human gastric adenocarcinoma cells in vitro and in vivo. Onco Targets Ther 2018; 11: 6705-6722 
[14] De Milito A, lessi E, Logozzi M et al. Proton pump inhibitors induce apoptosis of human B-cell tumors through a caspase-independent mechanism involving reactive oxygen species. Cancer Res 2007; 67 (11): 5408-5417

[15] Pamarthy S, Kulshrestha A, Katara GK et al. The curious case of vacuolar ATPase: regulation of signaling pathways. Mol Cancer 2018; 17 (1): 41. DOI: 10.1186/s12943-018-0811-3

[16] Udelnow A, Kreyes A, Ellinger S et al. Omeprazole inhibits proliferation and modulates autophagy in pancreatic cancer cells. PLoS One 2011; 6 (5): e20143

[17] Belouzard S, Millet JK, Licitra BN et al. Mechanisms of coronavirus cell entry mediated by the viral spike protein. Viruses 2012; 4: 1011-1033

[18] Mazzon M, Marsh M. Targeting viral entry as a strategy for broadspectrum antivirals. F1000Res. 2019; 8: F1000 Faculty Rev-1628. DOI: 10.12688/f1000research.19694.1

[19] Marshansky V, Futai M. The V-type $\mathrm{H}+$-ATPase in vesicular trafficking: targeting, regulation and function. Curr Opin Cell Biol 2008; 20 (4): $415-426$

[20] Touret F, Gilles M, Barral K et al. In vitro screening of a FDA approved chemical library reveals potential inhibitors of SARS-CoV-2 replication. bioRxiv. 2020; doi: 10.1101/2020.04.03.02384625

[21] Wang $\mathrm{H}$, Yang $\mathrm{P}$, Liu $\mathrm{K}$ et al. SARS coronavirus entry into host cells through a novel clathrin- and caveolae-independent endocytic pathway. Cell Res 2008; 18 (2): 290-301

[22] Sabolić I, Brown D, Verbavatz JM et al. (+ )-ATPases of renal cortical and medullary endosomes are differentially sensitive to Sch-28080 and omeprazole. Am J Physiol 1994; 266 (6 Pt 2): F868-F877

[23] Sachs G, Prinz C, Loo D et al. Gastric acid secretion: activation and inhibition. Yale J Biol Med 1994; 67 (3-4): 81-95

[24] Brecher M, Schornberg KL, Delos SE et al. Cathepsin cleavage potentiates the Ebola virus glycoprotein to undergo a subsequent fusion-relevant conformational change. J Virol 2012; 86: 364-372

[25] Sobhy $\mathrm{H}$. A review of functional motifs utilized by viruses. Proteomes 2016; 4 (1): 3

[26] Watanabe SM, Ehrlich LS, Strickland M et al. Selective targeting of virus replication by proton pump inhibitors. Sci Rep 2020; 10: 4003

[27] Tabata K, Arimoto M, Arakawa M et al. Unique Requirement for ESCRT factors in flavivirus particle formation on the endoplasmic reticulum. Cell Rep 2016; 16: 2339-2347

[28] Irie T, Licata JM, McGettigan JP et al. Budding of PPxY-containing rhabdoviruses is not dependent on host proteins TGS101 and VPS4A. J Virol 2004; 78: 2657-2665

[29] George SN, Garcha DS, Mackay A] et al. Human rhinovirus infection during naturally occurring COPD exacerbations. Eur Respir ] 2014; 44 (1): 87-96

[30] Long J, Wright E, Molesti E et al. Antiviral therapies against Ebola and other emerging viral diseases using existing medicines that block virus entry. Version 2. F1000Res 2015; 4: 30. DOI: 10.12688/f1000research.6085.2

[31] Sasaki T, Nakayama K, Yasuda H et al. A randomized, single-blind study of lansoprazole for the prevention of exacerbations of chronic obstructive pulmonary disease in older patients. J Am Geriatr Soc 2009; 57 (8): 1453-1457

[32] Casanova C, Baudet JS, del Valle Velasco M et al. Increased gastrooesophageal reflux disease in patients with severe COPD. Eur Respir J 2004; 23 (6): 841-845

[33] Su VY, Liao HF, Perng DW et al. Proton pump inhibitors use is associated with a lower risk of acute exacerbation and mortality in patients with coexistent COPD and GERD. Int J Chron Obstruct Pulmon Dis 2018; 13: 2907-2915

[34] Xiao F, Tang M, Zheng X et al. Evidence for Gastrointestinal Infection of SARS-CoV-2. Gastroenterology 2020; 158: 1831-1833.e3
[35] Aragão DS, Cunha TS, Andrade MCC et al. Purification and characterization of angiotensin converting enzyme 2 (ACE2) from murine model of mesangial cell in culture. Int J Biol Macromol 2011; 49: 79-84

[36] Liu J, Ji H, Zheng $W$ et al. Sex differences in renal angiotensin converting enzyme 2 (ACE2) activity are 17 $\beta$-oestradiol-dependent and sex chromosome-independent. Biol Sex Differ 2010; 1 (1): 6. DOI: 10.1186/2042-6410-1-6

[37] Farrell CP, Morgan M, Rudolph DS et al. Proton Pump Inhibitors Interfere With Zinc Absorption and Zinc Body Stores. Gastroenterol Res 2011; 4: 243-251

[38] Bojkova D, McGreig JE, McLaughlin K et al. SARS-CoV-2 and SARS-CoV differ in their cell tropism and drug sensitivity profiles. bioRxiv. 2020; doi:10.1101/2020.04.03.024257

[39] Shin JM, Kim N. Pharmacokinetics and pharmacodynamics of the proton pump inhibitors. J Neurogastroenterol Motil 2013; 19 (1): 25-35

[40] US Food and Drug Administration. Prilosec Label - FDA.https://www. accessdata.fda.gov/drugsatfda_docs/label/2008/022056s001019810s 087lbl.pdf, accessed on July 29 2020;

[41] Ozutemiz AO, Aydin $\mathrm{HH}$, Isler $\mathrm{M}$ et al. Effect of omeprazole on plasma zinc levels after oral zinc administration. Indian J Gastroenterol 2002; 21 (6): 216-218

[42] Reeves PG, O’Dell BL. Effects of dietary zinc deprivation on the activity of angiotensin-converting enzyme in serum of rats and guinea pigs. J Nutr 1986; 116: 128-134

[43] Speth R, Carrera E, Jean-Baptiste M et al. Concentration-dependent effects of zinc on angiotensin-converting enzyme-2 activity (1067.4). The FASEB Journal. 2014; 28 DOI: 10.1096/fasebj.28.1_supplement. 1067.4

[44] Cao JW, Duan SY, Zhang HX et al. Zinc deficiency promoted fibrosis via ROS and TIMP/MMPs in the myocardium of mice. Biol Trace Elem Res 2020; 196 (1): 145-152

[45] Homolak J, Kodvanj I. Widely available lysosome targeting agents should be considered as potential therapy for COVID-19. Int J Antimicrob Agents 2020; 106044 DOI: 10.1016/j.ijantimicag.2020.106044

[46] Blanc F, Waechter C, Vogel T et al. Interest of proton pump inhibitors in reducing the occurrence of COVID-19: A case-control study. Preprints. 2020; DOI: 10.20944/preprints202005.0016.v1

[47] World Health Organization R\&D Blueprint and COVID-19. 2020;https:// www.who.int/teams/blueprint/covid-19, accessed on July 29, 2020

[48] Almario CV, Chey WD, BMR Spiegel. Increased Risk of COVID-19 Among Users of Proton Pump Inhibitors. Am J Gastroenterol 2020; https://journals.Iww.com/ajg/Documents/AJG-20-1811_ R1(PUBLISH\%20AS\%20WEBPART).pdf, accessed on July 29, 2020

[49] Luxenburger $H$, Sturm L, Biever $P$ et al. Treatment with proton pump inhibitors increases the risk of secondary infections and ARDS in hospitalized patients with COVID-19: coincidence or underestimated risk factor? J Intern Med 2020; 10.1111/joim.13121 DOI: 10.1111/ joim. 13121

[50] Zhang X-Y, Wu H-B, Ling Y et al. Analysis of the effect of proton pump inhibitors on the course of common COVID-19. medRxiv. 2020; DOI: 10.1101/2020.06.07.20124776

[51] Taştemur Ş, Ataseven H. Is it possible to use Proton Pump Inhibitors in COVID-19 treatment and prophylaxis? Medical Hypotheses 2020; 110018. DOI: 10.1016/j.mehy.2020.110018

[52] Aguila EJT, Cua IHY. Repurposed GI Drugs in the Treatment of COVID-19. Dig Dis Sci 2020; 65 (8): 2452-2453 